\title{
Breast lymphatic drainage via the pulmonary lymphatic system
}

\author{
Thomas W. Barber • Michael S. Hofman • \\ Rodney J. Hicks
}

Received: 4 July 2010 /Accepted: 2 August 2010 /Published online: 7 September 2010

(C) UK Crown 2010

A 43-year-old woman underwent sentinel lymph node localisation prior to a left mastectomy and sentinel node biopsy for recurrent multifocal left-sided breast cancer. Previous treatment included a wide local excision and left axillary nodal clearance 15 months ago. Following one peri-areolar intradermal injection of $15 \mathrm{MBq}$ of ${ }^{99 \mathrm{~m}} \mathrm{Tc}$ antimony colloid in the upper inner quadrant of the left breast, dynamic planar imaging failed to identify a sentinel node. SPECT/CT imaging demonstrated a lymphatic channel extending from the left subpectoral region through the lingula of the left lung into the mediastinum posterior to the pulmonary trunk and terminating in the subcarinal nodal station (arrow). This unusual pattern of breast lymphatic drainage via the pulmonary lymphatic system is attributed to opening of collateral lymphatic channels following the previous left axillary nodal clearance. A left internal mammary sentinel lymph node (arrowhead) was also demonstrated and confirmed intraoperatively.

While it is commonly believed that spread of breast cancer beyond the regional lymph nodes is haematogenous, it is also increasingly being recognised that traditional anatomical descriptions of lymphatic anatomy do not necessarily reflect observed variations in lymphatic pathways $[1,2]$. Given that breast cancer is one of the known extrathoracic malignancies that metastasise to mediastinal nodes [3-5], this finding of a direct communication of

T. W. Barber $(\bowtie) \cdot$ M. S. Hofman $\cdot$ R. J. Hicks

Centre for Cancer Imaging, Peter MacCallum Cancer Centre,

7 St Andrews Place,

East Melbourne, Victoria, Australia 3002

e-mail: tomwbarber@yahoo.com.au

\section{S. Hofman}

e-mail: michael.hofman@petermac.org

R. J. Hicks

e-mail: rod.hicks@petermac.org breast lymphatic drainage with the intrapulmonary lymphatic system provides a potential mechanism for this pattern of spread. This case highlights how the combination of functional and anatomical information provided by SPECT/CT in lymphoscintigraphy continues to improve our understanding of potential paths of cancer spread.
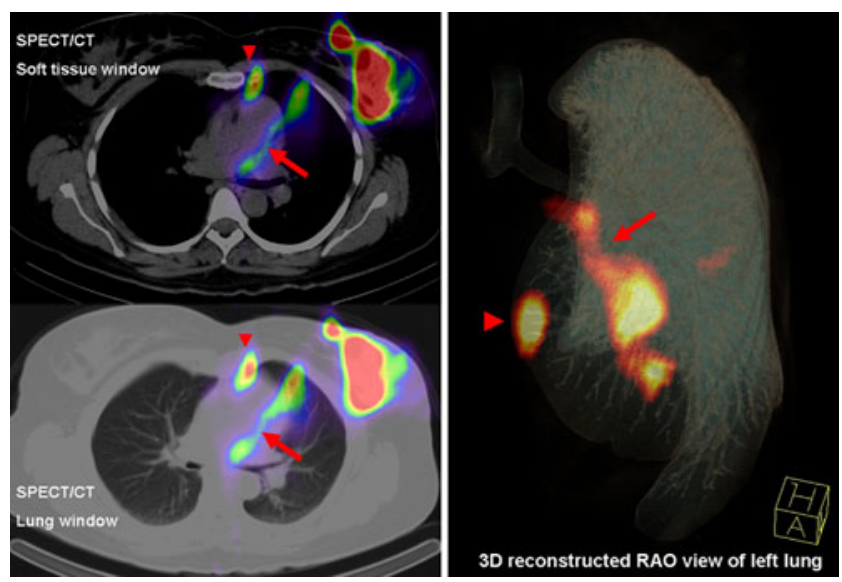

\section{References}

1. Topol M, Masłoń A. Some variations in lymphatic drainage of selected bronchopulmonary segments in human lungs. Ann Anat 2009;191(6):568-74.

2. Suami H, Pan WR, Mann GB, Taylor GI. The lymphatic anatomy of the breast and its implications for sentinel lymph node biopsy: a human cadaver study. Ann Surg Oncol 2008;15(3):863-71.

3. Riquet M, Berna P, Brian E, Badia A, Vlas C, Bagan P, et al. Intrathoracic lymph node metastases from extrathoracic carcinoma: the place for surgery. Ann Thorac Surg 2009;88(1):200-5.

4. Kamby C, Andersen J, Ejlertsen B, Birkler NE, Rytter L, Zedeler $\mathrm{K}$, et al. Pattern of spread and progression in relation to the characteristics of the primary tumour in human breast cancer. Acta Oncol 1991;30(3):301-8.

5. Thomas JM, Redding WH, Sloane JP. The spread of breast cancer: importance of the intrathoracic lymphatic route and its relevance to treatment. Br J Cancer 1979;40(4):540-7. 